\title{
Endocannabinoids as emerging suppressors of angiogenesis and tumor invasion (Review)
}

\author{
MAURIZIO BIFULCO ${ }^{1}$, CHIARA LAEZZA ${ }^{2}$, PATRIZIA GAZZERRO $^{1}$ and FRANCESCA PENTIMALLI ${ }^{3}$ \\ ${ }^{1}$ Dipartimento di Scienze Farmaceutiche, Università di Salerno, Via Ponte Don Melillo, 84084 Fisciano (SA); \\ ${ }^{2}$ Istituto di Endocrinologia ed Oncologia Sperimentale I.E.O.S., CNR; ${ }^{3}$ Dipartimento di Biologia e Patologia \\ Cellulare e Molecolare, Università di Napoli ‘Federico II', Via Pansini 5, 80131 Napoli, Italy
}

Received August 7, 2006; Accepted September 25, 2006

\begin{abstract}
The medicinal properties of extracts from the hemp plant Cannabis sativa have been known for centuries but only in the 90s membrane receptors for the Cannabis major principle were discovered in mammalian cells. Later on the endogenous ligands for the cannabinoid receptors were identified and the term 'endocannabinoid system' was coined to indicate the complex signaling system of cannabinoid receptors, endogenous ligands and the enzymes responsible for their biosynthesis and inactivation. The 'endocannabinoid system' is involved in a broad range of functions and in a growing number of pathological conditions. There is increasing evidence that endocannabinoids are able to inhibit cancer cell growth in culture as well as in animal models. Most work has focused on the role of endocannabinoids in regulating tumor cell growth and apoptosis and ongoing research is addressed to further dissect the precise mechanisms of cannabinoid antitumor action. However, endocannabinoids are now emerging as suppressors of angiogenesis and tumor spreading since they have been reported to inhibit angiogenesis, cell migration and metastasis in different types of cancer, pointing to a potential role of the endocannabinoid system as a target for a therapeutic approach of such malignant diseases. The potential use of cannabinoids to retard tumor growth and spreading is even more appealing considering that they show a good safety profile, regarding toxicity, and are already used in cancer patients as palliatives to stimulate appetite and to prevent devastating effects such as nausea, vomiting and pain.
\end{abstract}

\section{Contents}

1. Introduction

2. Cannabinoids and angiogenesis

Correspondence to: Dr Maurizio Bifulco, Dipartimento di Scienze Farmaceutiche, Università degli Studi di Salerno, Via Ponte Don Melillo, 84084 Fisciano (SA), Italy

E-mail:maubiful@unina.it,maubiful@unisa.it

Key words: endocannabinoids, angiogenesis, metastasis, cancer
3. Cannabinoids, cell migration, invasion and metastasis

4. Concluding remarks

\section{Introduction}

The recreational and therapeutic effects derived from the use of the hemp plant Cannabis sativa have been known for centuries and marijuana usage is still widely debated. Since the isolation of Cannabis major active principle, the $\Delta^{9}$-tetrahydrocannabinol (THC) (1), great progress has been made in understanding many of the pharmacological properties of cannabinoids. Following the cloning in mammalian cells of the first cannabinoid receptor several endogenous ligands, named 'endocannabinoids', were identified thereafter. Two major cannabinoid receptors, $\mathrm{CB} 1$ and $\mathrm{CB} 2$, both belonging to the class of $\mathrm{G}$ protein coupled receptors (GPCR) and several endocannabinoids such as anandamide (AEA) and 2arachidonoylglycerol (2-AG) have been identified and characterized. Together with the enzymes responsible for their synthesis, uptake and degradation, endocannabinoids and their receptors constitute the 'endocannabinoid system' which is involved in a variety of biological processes and is altered in a growing number of pathologies. In particular, there is increasing evidence that cannabinoid agonists are able to inhibit cancer cell growth in different types of cancer such as breast, colorectal, prostate, brain, skin and thyroid cancer (2).

The antineoplastic activity of THC was first described by Munson and colleagues (3), when neither cannabinoid receptors nor endocannabinoids had been discovered yet. The considerable progress obtained in cannabinoid research in the last decades has been extensively reviewed and cannabinoids have been suggested as potential anticancer agents $(4,5)$. This issue is still controversial since there are studies showing that cannabinoids can enhance tumor cell proliferation in some cellular contexts (2). Moreover, the undesired immunosuppressive effect triggered by CB2 agonists has to be taken into account. Most work has focused on the role of cannabinoids in regulating tumor cell growth and apoptosis, but it is worth noting that cannabinoids have an impact on other different mechanisms all contributing in cancer cells to the acquisition of a more aggressive phenotype: angiogenesis, cell migration, invasion and metastasis. 


\section{Cannabinoids and angiogenesis}

The hallmark of cancer cells is the acquired ability to proliferate despite the absence of mitogenic signals, but growth of solid tumors is dependent on the generation of new vascular supply. Therefore, targeting neoangiogenesis constitutes one of the most promising therapeutic approaches against cancer. Cannabinoids have been shown to inhibit angiogenesis in different models; in a mouse model of glioma, Blazquez and colleagues observed that blood vessel morphology was notably altered in tumors derived by either rat-C6-glioma cell or human grade IV astrocytoma cell inoculation, after cannabinoid treatment. Such tumors, following local administration of the CB2 receptor selective agonist JWH-133 (50 $\mu \mathrm{g} /$ day for 8 days or 25 days, respectively) were not only smaller in size but also had a paler appearance and a smaller, differentiated, impermeable vascular network compared to the controls. The authors showed that cannabinoid treatment was able to inhibit angiogenesis impairing vascular endothelial cell migration and survival and decreasing, in both C6-cell and human gliomas, the expression of proangiogenic factors such as vascular endothelial growth factor (VEGF), angiopoietin-2 and matrix metalloproteinase-2 (MMP2), a proteolytic enzyme involved in tissue remodeling during angiogenesis and metastasis (6).

Moreover, a recent cDNA array-based study (7) showed that JWH-133 treatment of mice-bearing gliomas downregulates the expression of several VEGF pathway-related genes, such as VEGF itself and the hypoxia-inducible factor- $1 \alpha$ (HIF-1 $\alpha$ ) a master transcription factor for oxygen homeostasis regulation, with a key role in angiogenesis and in VEGF gene expression regulation (8). VEGF production was inhibited in glioma cell lines and in tumor cells obtained from a human glioblastoma multiforme (GBM) biopsy specimen by treatment with the mixed CB1/CB2 agonist WIN-55,212-2 (100 nM). In glioma cells, also anandamide $(2 \mu \mathrm{M})$ and JWH-133 (100 nM) were as effective in inhibiting VEGF production. Moreover, both CB1 and CB2 antagonists (SR141716, 0.5 $\mu \mathrm{M}$ and SR144528, $0.5 \mu \mathrm{M}$ ) prevented WIN-55,212-2 action in these cells indicating a role for $\mathrm{CB}$ receptors in VEGF inhibition. Accordingly, treatment of C6 glioma cells with WIN-55,212-2 or JWH-133 resulted in decreased activation of the VEGF receptor VEGFR-2, and this effect could be inhibited by pharmacological blockade of ceramide biosynthesis. Consistently, intra-tumoral administration of THC to two patients affected by GBM was reported to decrease both the VEGF level and VEGFR-2 activation in the tumors (7). The two patients were enrolled in a phase III clinical trial aimed to assess THC effect on recurrent GBM.

VEGF is one of the major key players in angiogenesis acting as a potent stimulator of new vessel formation and many tumors are reported to have increased VEGF expression compared to their normal tissue counterparts (9); in certain cell types the ras oncogene activation is responsible for the VEGF up-regulation (10). This is consistent with the finding that the metabolic stable anandamide analogue, 2-methylarachidonyl-2'-fluoro-ethylamide (Met-F-AEA), inhibits the growth in vivo of established thyroid tumors by reducing p21ras activity (11) and both VEGF and its receptor Flt-1 (VEGFR-1) expression (12).
Cannabinoid agonists have also been shown to inhibit in vivo skin tumor growth and angiogenesis in gliomas and established thyroid tumors. Both WIN-55,212-2 and JWH-133 were able to inhibit the growth of tumors obtained in mice upon inoculation of highly malignant PDV.C57 epidermal cancer cells. Accordingly, cannabinoid treatment induced impaired tumor vascularization reducing blood vessel size and decreasing the expression of proangiogenic factors such as VEGF, placental growth factor (PLGF), and angiopoietin 2 (13).

Recently, a decrease in VEGF expression level following treatment with 1-10 $\mu \mathrm{M}$ of WIN-55,212-2 has been reported also in the prostate carcinoma cell line LNCap. In vitro, cannabinoid agonists treatment was able to inhibit LNCap cell growth in a manner dependent on $\mathrm{CB}$ receptors since both SR141716 and SR144528 could partially reverse WIN55,212-2 effect (14).

VEGF released from cancer cells exerts a role as an autocrine-paracrine factor able not only to stimulate the proliferation of endothelial cells and the formation of new vessels, but also the migration and spreading of cancer cells. Therefore, the down-regulation of both VEGF and its receptors induced in vivo by (endo)cannabinoids may also lead to a direct effect not only on tumor neoangiogenesis but also on growth and metastasis of cancer cells.

\section{Cannabinoids, cell migration, invasion and metastasis}

During neoplastic progression some cancer cells acquire the ability of escaping the primary tumor mass. Such malignant cells invade surrounding tissues and migrate to distant sites where they may found new colonies; metastases, form in most types of human cancer and are responsible for most cancer deaths (9) (Fig. 1).

Several works indicate that cannabinoids play a role in cell migration, invasion and metastasis. Recently, cannabidiol (CBD), a non-psychoactive cannabinoid, has been shown to inhibit not only cell growth (15) but also cell migration of the U87 human glioma cells in a dose-dependent manner. The mechanism of such inhibition has to be further investigated but it seems not to involve classical $\mathrm{CB}$ receptors or $\mathrm{G}$ proteincoupled signaling (16). Nithipatikom and colleagues reported that increasing levels of the endocannabinoid 2-AG, by blocking its metabolism, could significantly inhibit invasion of the human androgen-independent prostate cancer cells, PC-3 and DU-145. Also, WIN55,212-2 and (R)-(+)-methanandamide were able to inhibit, in a concentration-dependent manner, PC-3 and DU-145 cell invasion and this effect involved the CB1 receptor pathway (17). Furthermore, the authors tested several compounds able to inhibit 2-AG hydrolysis and prostate cancer cell invasion and proposed them as a potential therapeutic approach for this disease (18).

Accordingly, anandamide also showed a potent antiproliferative and cytotoxic effect on metastatic prostatic cancer cells (19). Several studies indicate a THC-mediated antitumor immune response $(2,20)$; however, anandamide has been shown to inhibit chemokine-induced migration of SW480 colon carcinoma cells and of CD8 ${ }^{+} \mathrm{T}$ lymphocytes via $\mathrm{CB} 1$ or $\mathrm{CB} 2$ receptor, respectively, suggesting that, at least 

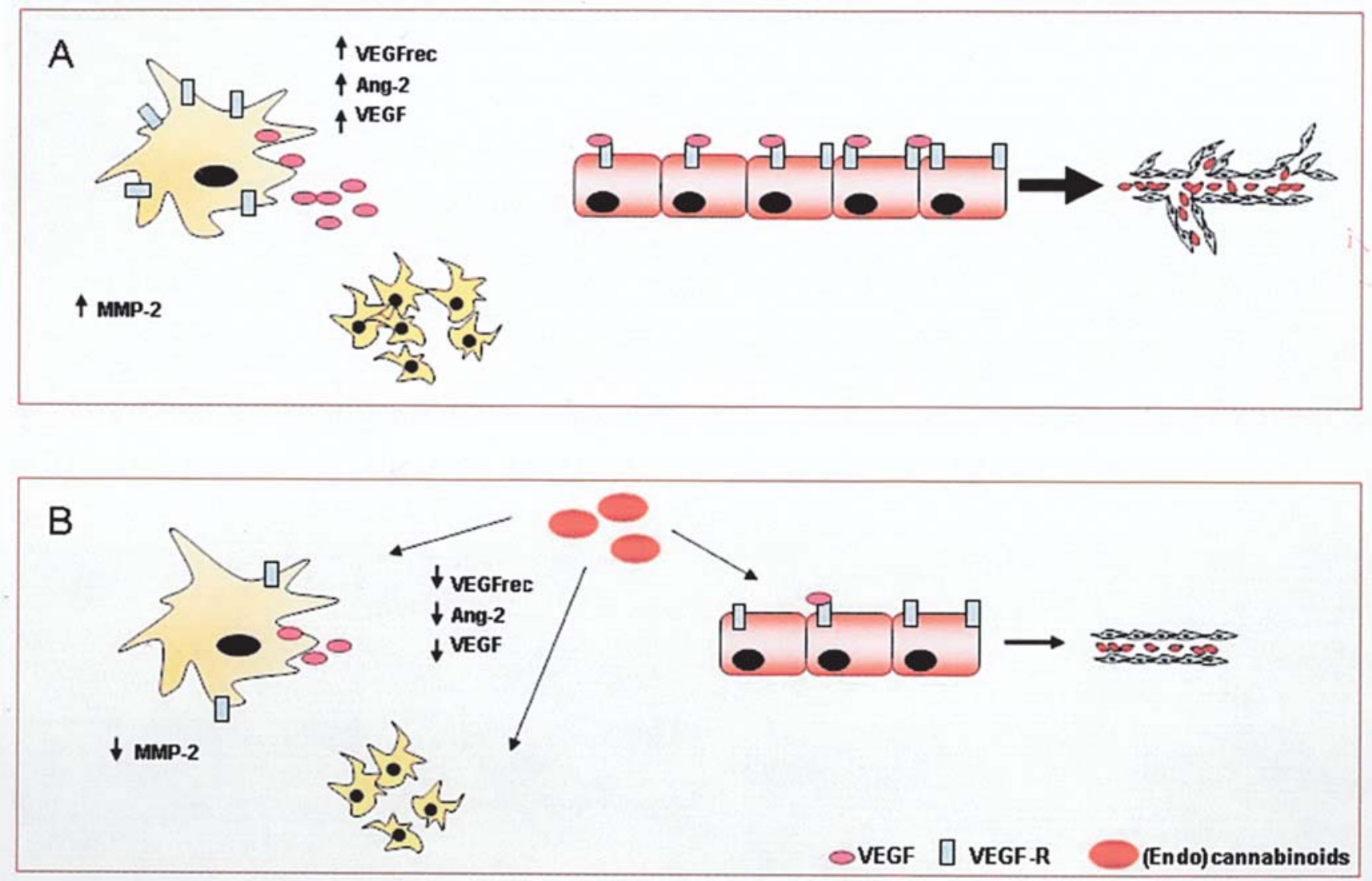

Figure 1. (Endo)cannabinoids in angiogenesis and metastasis. A schematic representation of the principal mechanisms modulated by (endo)cannabinoids during tumor progression. (A) Tumor, metastatic and vascular endothelial cells (from left to right) during neo-angiogenesis and tumor cell spreading when VEGF, VEGF-R, Ang-2 and MMP-2 are generally up-regulated. (B) (Endo)cannabinoid effects on tumor, metastatic and vascular endothelial cells in the control of these processes: VEGF, VEGF-R, Ang-2 and MMP-2 are all down-regulated resulting in reduced angiogenesis, cancer cell migration and metastasis.

in colon cancer, a specific inhibition of tumor cell migration could be achieved without affecting the immune system (21).

Blazquez and colleagues also reported that treatment with WIN-55,212-2 (25 nM) or JWH-133 (25 nM) could directly inhibit cell migration of the vascular endothelial cells HUVECs. WIN-55,212-2 effect, assessed by the Boyden chamber method, was prevented by both SR141716 $(0.5 \mu \mathrm{M})$ and SR144528 $(0.5 \mu \mathrm{M})$ pointing to the involvement of CB receptors in HUVEC cell migration. Targeting vascular endothelial cell migration constitutes another feasible strategy for cannabinoids to inhibit angiogenesis (6).

Endocannabinoid-induced antitumor action was observed also in other tissues. Met-F-AEA treatment was able to suppress proliferation of the metastasis-derived thyroid cancer cell line MPTK-6 more efficaciously with respect to the primary thyroid cancer-derived TK- 6 cell line likely due to a stronger up-regulation of the $\mathrm{CB} 1$ receptor in the former. These data were further supported by using an in vivo model of metastatic spreading such as the formation of lung nodules upon inoculation of Lewis lung carcinoma cells. In this system, Met-F-AEA treatment resulted in a dramatic reduction in both the number and the size of metastatic nodules and this effect was totally reversed by the $\mathrm{CB} 1$ receptor antagonist SR141716A (12).
Additionally, Met-F-AEA treatment is able to inhibit both adhesion and migration of the highly invasive and metastatic breast cancer cell lines, MDA-MB-231 and TSA-E1, as tested in in vitro adhesion and migration assays with type IV collagen, the major component of the basement membrane. Also, this effect is mediated by the $\mathrm{CB} 1$ receptor, since it is antagonized by SR141716A, and is accompanied by a remarkable decrease in SRC and focal adhesion kinase (FAK) phosphorylation, two tyrosine kinases involved in the loss of adhesion and invasion. Furthermore, Met-F-AEA treatment significantly reduced the number and dimension of metastatic nodes induced by injection of TSA-E1 cells in syngenic mice (22).

\section{Concluding remarks}

Given the latest additions in (endo)cannabinoids research it is emerging that these compounds have the ability of modulating cancer progression. Angiogenesis, in particular, is a prerequisite for the progression of most solid growth and much effort has been directed to the development of agents capable of disrupting this process. However, this strategy has not been significant in clinical practice and further research is ongoing to increase efficacy of such an approach (8). Cannabinoids have been shown to inhibit vascular 
endothelial cell migration and to down-regulate crucial angiogenic factors in different types of tumors, such as skin cancer, one of the most common malignancies in human (13), or glioma, one of the most malignant forms of cancer with a 1-2 years rate of survival following diagnosis (7). VEGF/VEGF receptor pathway represents a key player in tumor-induced angiogenesis and, consequently, tumor progression and metastasis. The humanized anti-VEGF monoclonal antibody bevacizumab has been recently approved for cancer treatment and results are encouraging even if not untroubled $(23,24)$. Endocannabinoids warrant further investigation since they have been clearly shown to interfere with the VEGF pathway in different systems and may provide a useful tool to target VEGF in cancer. Moreover, the invasive and metastatic stages of cancer progression correlate with poor prognosis and unsuccessful clinical treatment and (endo)cannabinoids have been shown to play a role in these processes; (endo)cannabinoids inhibited cell migration of glioma, colon carcinoma cells and breast cancer cells; inhibited invasion of androgen-independent prostate cancer and metastatic spreading of thyroid cancer and Lewis lung carcinoma cells.

Therefore, even if further basic and preclinical research are required to clarify the molecular mechanisms of cannabinoids action in cancer and to test their effectiveness in patients, targeting the endocannabinoid system represents a very promising therapeutic approach for the treatment of different types of cancer. Cannabinoids have the great advantage of being well tolerated, regarding toxicity, and of having such remarkable palliative effects in cancer patients that their use has already been approved for clinical practice (5).

\section{Acknowledgements}

This work was supported by the Associazione Educazione e Ricerca Medica Salernitana (ERMES).

\section{References}

1. Gaoni Y and Mechoulam R: Isolation, structure and partial synthesis of an active constituent of hashish. J Am Chem Soc 86: 1646-1647, 1964

2. Bifulco M, Laezza C, Pisanti S and Gazzerro P: Cannabinoids and cancer: pros and cons of an antitumor strategy. Br J Pharmacol 148: 123-135, 2006.

3. Munson AE, Harris LS, Friedman MA, Dewey WL and Carchman RA: Antineoplastic activity of cannabinoids. J Natl Cancer Inst 55: 597-602, 1975.

4. Bifulco M and Di Marzo V: Targeting the endocannabinoid system in cancer therapy: a call for further research. Nat Med 8: 547-550, 2002.

5. Guzman M: Cannabinoids: potential anticancer agents. Nat Rev Cancer 3: 745-755, 2003.

6. Blazquez C, Casanova ML, Planas A, Del Pulgar TG, Villanueva C, Fernandez-Acenero MJ, Aragones J, Huffman JW, Jorcano JL and Guzman M: Inhibition of tumor angiogenesis by cannabinoids. FASEB J 17: 529-531, 2003.
7. Blazquez C, Gonzalez-Feria L, Alvarez L, Haro A, Casanova ML and Guzman M: Cannabinoids inhibit the vascular endothelial growth factor pathway in gliomas. Cancer Res 64: 5617-5623, 2004

8. Strieter RM: Masters of angiogenesis. Nat Med 11: 925-927, 2005.

9. Hanahan D and Weinberg RA: The hallmarks of cancer. Cell 100: 57-70, 2000.

10. Rak J, Mitsuhashi Y, Sheehan C, Tamir A, Viloria-Petit A, Filmus J, Mansour SJ, Ahn NG and Kerbel RS: Oncogenes and tumor angiogenesis: differential modes of vascular endothelial growth factor up-regulation in ras-transformed epithelial cells and fibroblasts. Cancer Res 60: 490-498, 2000.

11. Bifulco M, Laezza C, Portella G, Vitale M, De Petrocellis L, Orlando P and Di Marzo V: Control by the endogenous cannabinoid system of ras oncogene-dependent tumor growth. FASEB J 15: 2745-2747, 2001

12. Portella G, Laezza C, Laccetti P, De Petrocellis L, Di Marzo V and Bifulco M: Inhibitory effects of cannabinoid CB1 receptor stimulation on tumor growth and metastatic spreading: actions on signals involved in angiogenesis and metastasis. FASEB J 17: 1771-1773, 2003.

13. Casanova ML, Blazquez C, Martinez-Palacio J, Villanueva C, Fernandez-Acenero MJ, Huffman JW, Jorcano JL and Guzman M: Inhibition of skin tumor growth and angiogenesis in vivo by activation of cannabinoid receptors. J Clin Invest 111: 43-50, 2003.

14. Sarfaraz S, Afaq F, Adhami VM and Mukhtar H: Cannabinoid receptor as a novel target for the treatment of prostate cancer. Cancer Res 65: 1635-1641, 2005.

15. Massi P, Vaccani A, Ceruti S, Colombo A, Abbracchio MP and Parolaro D: Antitumor effects of cannabidiol, a non-psychoactive cannabinoid, on human glioma cell lines. J Pharmacol Exp Ther 308: 838-845, 2004

16. Vaccani A, Massi P, Colombo A, Rubino T and Parolaro D: Cannabidiol inhibits human glioma cell migration through a cannabinoid receptor-independent mechanism. Br J Pharmacol 144: 1032-1036, 2005.

17. Nithipatikom K, Endsley MP, Isbell MA, Falck JR, Iwamoto Y, Hillard CJ and Campbell WB: 2-Arachidonoylglycerol: a novel inhibitor of androgen-independent prostate cancer cell invasion. Cancer Res 64: 8826-8830, 2004.

18. Nithipatikom K, Endsley MP, Isbell MA, Wheelock CE, Hammock BD and Campbell WB: A new class of inhibitors of 2-arachidonoylglycerol hydrolysis and invasion of prostate cancer cells. Biochem Biophys Res Commun 332: 1028-1033, 2005.

19. Mimeault M, Pommery N, Wattez N, Bailly C and Henichart JP: Anti-proliferative and apoptotic effects of anandamide in human prostatic cancer cell lines: implication of epidermal growth factor receptor down-regulation and ceramide production. Prostate 56: $1-12,2003$.

20. McKallip RJ, Nagarkatti M and Nagarkatti PS: Delta-9-tetrahydrocannabinol enhances breast cancer growth and metastasis by suppression of the antitumor immune response. J Immunol 174: 3281-3289, 2005.

21. Joseph J, Niggemann B, Zaenker KS and Entschladen F: Anandamide is an endogenous inhibitor for the migration of tumor cells and $\mathrm{T}$ lymphocytes. Cancer Immunol Immunother 53: 723-728, 2004.

22. Grimaldi C, Pisanti S, Laezza C, Malfitano AM, Santoro A, Vitale M, Caruso MG, Notarnicola M, Iacuzzo I, Portella G, Di Marzo V and Bifulco M: Anandamide inhibits adhesion and migration of breast cancer cells. Exp Cell Res 312: 363-373, 2006.

23. Ferrara N, Hillan KJ and Novotny W: Bevacizumab (Avastin), a humanized anti-VEGF monoclonal antibody for cancer therapy. Biochem Biophys Res Commun 333: 328-335, 2005.

24. Neri D and Bicknell R: Tumour vascular targeting. Nat Rev Cancer 5: 436-446, 2005. 